\title{
Survival Analysis of Stage II Gastric Cancer Patients after D2 Gastrectomy: A Chinese People-based Research Running Head: Survival of Stage II Gastric Cancer
}

\section{Zi-Jian Deng}

Guangdong Provincial Key Laboratory of Colorectal and Pelvic Floor Diseases, The Sixth Affiliated Hospital, Sun Yat-Sen University

Run-Cong Nie

Sun Yat-sen University Cancer Center

Jun Lu

Fujian Medical University Union Hospital

\section{Xi-Jie Chen}

Sixth Affiliated Hospital of Sun Yat-sen University

Jun Xiang

Sixth Affiliated Hospital of Sun Yat-sen University

Chang-ming Huang

Fujian Medical University Union Hospital

\section{Ying-bo Chen}

Sun Yat-sen University Cancer Center

\section{Jun-Sheng Peng}

Sixth Affiliated Hospital of Sun Yat-sen University

Shi Chen ( $\nabla$ chensh47@mail.sysu.edu.cn )

Sixth Affiliated Hospital of Sun Yat-sen University

\section{Research Article}

Keywords: gastric cancer, Stage II, Prognosis, adjuvant chemotherapy, Propensity score matching

Posted Date: January 25th, 2021

DOl: https://doi.org/10.21203/rs.3.rs-143889/v1

License: (c) (i) This work is licensed under a Creative Commons Attribution 4.0 International License. Read Full License 



\section{Survival analysis of stage II gastric cancer patients after D2 gastrectomy: A Chinese people-based research}

\section{Running head: Survival of stage II gastric cancer}

Zi-Jian Deng ${ }^{1,2 \#} ;$ Run-Cong Nie ${ }^{3 \#} ;$ Jun-Lu ${ }^{4 \#}$, Xi-Jie Chen ${ }^{1,2}$; Jun Xiang ${ }^{1,2}$; Chang-Ming Huang ${ }^{4}$ Y Ying-Bo Chen ${ }^{3}$; Jun-Sheng Peng ${ }^{1,2 *}$ Shi Chen ${ }^{1,2^{*}}$

1. Guangdong Provincial Key Laboratory of Colorectal and Pelvic Floor Diseases, The Sixth Affiliated Hospital, Sun Yat-Sen University, Guangzhou, Guangdong 510655, P. R. China.

2. Department of Gastrointestinal Surgery, The Sixth Affiliated Hospital, Sun Yat-Sen University, Guangzhou, 510655, P. R. China.

3. Department of Gastric Surgery, Sun Yat-sen University Cancer Center; State Key Laboratory of Oncology in South China; Collaborative Innovation Center for Cancer Medicine, Guangzhou, China

4. Department of Gastric Surgery, Fujian Medical University Union Hospital, No. 29 Xinquan Road, Fuzhou, 350001, P.R. China.

\#: These three authors contributed equally to this manuscript.

Supported by Guangzhou Science and Technology Project (grant number 201803010040) and Nation Key Clinical Discipline.

\section{"Correspondence to:}

Jun-Sheng Peng: The Sixth Affiliated Hospital, Sun Yat-Sen University, No 26, Yuancun Erheng Road, Tianhe District, 510655, Guangzhou, P. R. China. 
Phone: +86.20.3825.4092; E-mail: pengjsh@mail.sysu.edu.cn

Shi Chen: The Sixth Affiliated Hospital, Sun Yat-Sen University, No 26, Yuancun Erheng Road, Tianhe District, 510655, Guangzhou, P. R. China.

Phone: +86.20.3825.4092; E-mail: chensh47@mail.sysu.edu.cn 


\section{Abstract}

Objective: The benefit of adjuvant chemotherapy is still controversial for stage II gastric cancer patients. This study aims to identify prognostic factors to guide individualized treatment for stage II gastric cancer patients.

Methods: We retrospectively reviewed 1121 stage II gastric cancer patients who underwent D2 radical gastrectomy from 2007-2017 in the Sixth Affiliated Hospital of Sun Yat-sen University, FuJian Medical School Affiliated Union Hospital and Sun Yat-sen University Cancer Center. Propensity score matching (PSM) was used to ensure that the baseline data were balanced between the adjuvant chemotherapy (AC) group and surgery-only group. Kaplan-Meier survival and multivariate Cox regression analyses were carried out to identify independent prognostic factors.

Results: In univariate analysis, after propensity score matching, age, tumor location, tumor size, CEA, T stage and $\mathrm{N}$ stage were associated with overall survival (OS). Multivariate analysis illustrated that age $\geq 60$ years old, linitis plastica and T4 were independent risk factors for OS, but lower location and adjuvant chemotherapy were protective factors.

Conclusion: Adjuvant chemotherapy is helpful for stage II gastric cancer patients. These prognostic factors can help guide individual therapy.

Key words: gastric cancer, Stage II, Prognosis, adjuvant chemotherapy, Propensity score matching. 


\section{Introduction}

Despite the rapid advances that continue to improve comprehensive therapy and screening methods, gastric cancer is still the fourth most common malignant tumor in the world $(989,600$ new cases per year) and the second leading cause of death among all malignant tumors $(738,000 \text { deaths annually })^{1}$. Approximately $60 \%$ of new cases occur in three eastern Asian countries: China, Japan and South Korea ${ }^{2}$. Although the incidence of gastric cancer has decreased worldwide since the 1950s, China still accounts for $42.6 \%$ of the global incidence and approximately $45 \%$ of gastric cancer-related deaths s.4 $^{3,4}$

In recent years, adjuvant chemotherapy has been widely used for stage II and III gastric cancer patients to improve the 5-year overall survival rate. The ACTS-GC study, a randomized phase III trial, showed that the overall survival rate at 5 years was $71.7 \%$ in the adjuvant group and $61.1 \%$ in the surgery-only group (HR [95\%Cl], 0.669 [0.540-0.828] $)^{5}$. Another famous study in Asia, the CLASSIC study, also reported a hazard ratio for 5-year overall survival of 0.66 $(95 \% \mathrm{Cl}, 0.51-0.85 ; p=0.0015)$ for surgery and adjuvant chemotherapy with capecitabine and oxaliplatin for 6 months after a median follow-up of 62.4 months ${ }^{6}$. These results from randomized controlled trials provided hard evidence of the survival benefits associated with adjuvant chemotherapy. However, the subgroup analysis of 3-year disease-free survival in the CLASSIC study demonstrated that the improvement in the adjuvant chemotherapy group for stage II disease was not as evident as that for stage IIla or IIlb disease. The 
3-year disease-free survival rates for stage II disease were 85\% (adjuvant chemotherapy group) vs $71 \%$ (surgery-only group), those for stage Illa disease were $66 \%$ vs $51 \%$, and those for stage IIIb disease were $61 \%$ vs $33 \%^{7}$. Some clinics considered that adjuvant chemotherapy for all stage II or III gastric cancer patients may be unnecessary or even harmful to some patients ${ }^{8}$. Furthermore, Choi et al. confirmed that stage II or III gastric cancer patients with high microsatellite instability might not benefit from adjuvant chemotherapy ${ }^{9}$. Some researchers have tried to use biomarkers to determine the necessity of chemotherapy, but all of these studies were retrospective, and the results were unsatisfactory.

Therefore, there is still no evaluation system for stage II gastric cancer patients to determine if adjuvant chemotherapy is needed. Thus, the purpose of this study is to confirm the role of adjuvant chemotherapy in the treatment of stage II gastric cancer and determine some prognostic factors for the establishment of a prediction model to guide individualized treatment for stage II gastric cancer.

\section{Materials and methods}

\section{Ethics statement}

This study was conducted in accordance with the ethical standards of the World Medical Association Declaration of Helsinki and the Ethical Guidelines for Clinical Research. The protocol was approved by the ethics committee of 
Sixth affiliated hospital of Sun Yat-sen University; Fujian Medical University Union Hospital and Sun Yat-sen University cancer center. Informed consent was provided by all patients.

\section{Patients}

We retrospectively collected clinicopathological factors from 1389 gastric cancer patients from the Sixth Affiliated Hospital of Sun Yat-sen University, FuJian Medical School Affiliated Union Hospital and Sun Yat-sen University Cancer Center who underwent D2 radical gastrectomy alone or radical surgery followed by adjuvant chemotherapy. We applied inclusion and exclusion criteria to select eligible patients. The inclusion criteria were as follows: 1) pathologically confirmed stage II gastric adenocarcinoma (according to the $8^{\text {th }}$ TNM staging system of the American Joint Committee on Cancer), 2) D2 radical gastrectomy, 3) enough clinicopathological factors to analyze, 4) no synchronized tumors, and 5) complete follow-up data. The exclusion criteria were as follows: 1) previous neoadjuvant chemotherapy, immunotherapy or radiotherapy, 2) missing important clinicopathological factor information, 3) incomplete follow-up information, 4) severe hepatic and renal insufficiency, and 5) age younger than 18 years. Finally, a total of 1121 patients with stage II gastric cancer were selected, with 805 (71.8\%) patients in the adjuvant chemotherapy (AC) group and $316(28.2 \%)$ in the surgery-only group.

\section{Variables}


The continuous variables were turned into suitable categorical variables. The analyzed variables included age $(<60, \geq 60$ years old), sex (male, female), carcinoembryonic antigen (CEA) $(<5, \geq 5 \mu \mathrm{g} / \mathrm{L})$, carbohydrate antigen 19-9 (CA199) (<37, $\geq 37 \mathrm{U} / \mathrm{ml})$, hemoglobin (HB) $(<120, \geq 120 \mathrm{~g} / \mathrm{L})$, primary tumor location (upper, middle, lower or linitis plastica), primary tumor size $(<5, \geq 5$ $\mathrm{cm}$ ), $\mathrm{T}$ stage (T1/2/3, T4), $\mathrm{N}$ stage (negative, positive), tumor histology (mucinous adenocarcinoma or signet cell carcinoma), and adjuvant chemotherapy. The cut-off points for age and tumor size were classified by the "x-tile" program. The cut-off values of CEA, CA199 and HB were taken as the reference standards. T stage and $\mathrm{N}$ stage were identified by the postoperative pathological report. Poorly differentiated adenocarcinoma, including mucinous adenocarcinoma and signet cell carcinoma, were separated from gastric adenocarcinoma for the analysis.

\section{Statistical analysis}

Descriptive statistics were used to summarize distributions of the variables, and the chi-square test was used to evaluate the baseline categorical variables. Then, age was used as a covariate for propensity score matching (PSM). After 1:2 propensity score matching, the baseline clinicopathologic characteristics and overall survival (OS) were analyzed. OS was calculated from the date of surgery to death from cancer-related causes. The Kaplan-Meier method was used to draw survival curves, and the differences among curves were compared by log-rank test. Next, the variables 
with a $p$ value $<0.10$ in univariate analysis were enrolled in multivariate analysis. Multivariate Cox regression was used to determine independent prognostic factors associated with overall survival, and the final prognostic factors were selected by the stepwise method. A two-sided $p$ value $<0.05$ was considered significant in all analyses. All analyses were carried out by SPSS v.22.0 (SPSS, Inc., Chicago, IL, USA).

\section{Propensity score matching}

The patients were not randomly distributed into the adjuvant chemotherapy group or surgery-only group, which contributed to selection bias, so PSM was used to control selection bias and balance unbalanced covariates associated with the outcome. In this study, 1:2 nearest-neighbor matching for PSM without replacement was utilized. The caliper width was 0.05 .

\section{Result}

\section{Patient characteristics}

The clinicopathological factors of both group stage II gastric cancer patients are shown in Table 1. Before propensity score matching, the distribution of age $(p=0.002)$ and signet ring cell carcinoma $(p=0.007)$ were evidently different between the two groups. Compared with the surgery-only group, the AC group had fewer patients aged $\geq 60$ years (48.4\% vs $58.9 \%)$ but more patients with signet ring cell carcinoma (18.0\% vs $11.4 \%)$. Moreover, there were no differences in sex, CEA, CA199, HB, site of primary tumor, size 
of primary tumor, $\mathrm{T}$ stage, $\mathrm{N}$ stage or mucinous adenocarcinoma between groups. To avoid overmatching, we selected age as a covariate to estimate the propensity score because age was associated with OS $(p<0.001$, Table2). After 1:2 PSM, all variates were balanced $(p>0.05)$ except for signet ring cell carcinoma $(p=0.038)$. In total, 948 patients were selected by PSM, including 632 patients with adjuvant chemotherapy and 316 patients without adjuvant chemotherapy.

\section{Univariate survival analysis}

The prognoses of the AC group and surgery-only group were compared by Kaplan-Meier survival analysis. Before PSM, the survival curves illustrated that the prognosis of the $\mathrm{AC}$ group was significantly better than that of the surgery-only group $(p=0.034)$. The mean overall survival time of the $A C$ group was $107.48 \pm 1.89$ months, which was longer than that of the surgery-only group (95.53 \pm 3.06 months). However, we amazingly found that after PSM, the difference in overall survival curves between the two groups was not as significant as before $(p=0.060)$. The mean overall survival time was 107.18 \pm 2.12 months (AC) vs $95.53 \pm 3.06$ months (surgery-only) (Fig. 1).

Furthermore, the results also revealed that age $(p<0.001)$, primary tumor location $(p<0.001)$, primary tumor size $(p<0.001)$, CEA $(p=0.014)$, T stage $(p=0.009)$ and $N$ stage $(p=0.006)$ were obviously associated with OS(Table 2 \&Fig.2), but CA199 ( $p=0.143), H B(p=0.206)$, mucinous adenocarcinoma 
$(p=0.740)$ and signet ring cell carcinoma $(p=0.164)$ had statistically insignificant associations (Table 2). The results of the above variables were similar after PSM (Table 2\& Fig.3). Regarding sex, female patients had better survival than male patients before PSM $(p=0.025)$. However, the differences in OS between sexes were not significantly after PSM ( $P=0.075)$ (Fig4).

\section{Multivariate Cox regression analyses}

Cox proportional hazard models were established to identify independent prognostic factors for overall survival after matching. The results demonstrated that old age, high T stage and linitis plastica were independent risk factors for poor survival, while lower primary tumor and adjuvant chemotherapy were protective factors (Table 3).

\section{Discussion}

Although the incidence of gastric cancer is declining worldwide ${ }^{10}$, most gastric cancer patients are diagnosed at an advanced stage in China, so D2 radical gastrectomy and adjuvant chemotherapy still play a critical role in gastric cancer treatment ${ }^{4}$. Many important clinical studies have confirmed the significance of adjuvant chemotherapy, including the INT-0116 study ${ }^{11}$, MAGIC study $^{12}$, CLASSIC study ${ }^{6,7}$ and ACTS-GC study ${ }^{5,13}$. However, most patients in the INT-0116 study underwent D0 or D1 gastrectomy (a total of 90\%). Likewise, D2 radical gastrectomy was not used as standard surgery in the MAGIC study. 
Therefore, some Asian doctors doubt that these results are appropriate for gastric cancer patients because D2 radical gastrectomy has been the standard of care in east Asia ${ }^{14,15}$. In addition, the CLASSIC study and ACTS-GC study have confirmed the positive effects of adjuvant chemotherapy after D2 gastrectomy, but their inclusion criteria were based on the 6th edition AJCC and 2nd edition Japanese Gastric Cancer Association (JGCA) guidelines, respectively. As the CLASSIC study showed, the improvement in 3-year disease-free survival was not as evident for patients with stage II disease as it was for those with stage III disease in the adjuvant chemotherapy group. In the JCOG8801 study ${ }^{16}$, patients with T1-2N0/+ disease were considered to gain no survival benefits from adjuvant chemotherapy. Therefore, whether stage II gastric cancer patients need adjuvant chemotherapy and what kind of patients can benefit from adjuvant chemotherapy remain to be identified. In this study, we used multicenter data in China to explore this question.

The benefits of adjuvant chemotherapy for stage III gastric cancer has been widely demonstrated. However, these benefits remain controversial for stage II gastric cancer ${ }^{16-18}$. Recently, Yuming Jiang et al. ${ }^{8}$ reported some prognostic risk factors and tried to predict the survival benefit of adjuvant chemotherapy for patients with stage II and stage III gastric cancer, but no subgroup analysis was carried out in their study. In addition, although many studies have identified a series of prognostic factors related to gastric cancer ${ }^{19-21}$, none of the studies discussed stage II gastric cancer separately. Thus, in our study, we attempted 
to use some available and typical clinicopathological factors and identify their effects on the survival of stage II gastric cancer patients, with the aim to build a model for selecting patients with stage II gastric cancer who can benefit from adjuvant chemotherapy.

Our study demonstrated that age $\geq 60$ years, linitis plastica, and T4 were independent prognostic factors in stage II gastric cancer patients. Contrary to the results of other similar studies ${ }^{22,23}, \mathrm{~N}$ stage was not a prognostic factors in the results of the multivariate analysis. Additionally, patients with lymph node metastasis had better OS (mean OS, $104.76 ; 95 \% \mathrm{Cl}, 100.73$ to 108.80 ) than those without lymph node metastasis (mean OS, $100.08 ; 95 \% \mathrm{Cl}, 94.77$ to 105.40). In other studies, patients classified as stage II/III or all stages were included. However, stage II (according to 8th edition AJCC guidelines) gastric cancer is a special stage. Stage II gastric cancer patients with a late T stage usually have early $\mathrm{N}$ stage disease. Our results confirmed that $\mathrm{T}$ stage is more meaningful than $\mathrm{N}$ stage in influencing the prognosis of stage II gastric cancer patients. Therefore, due to the late $\mathrm{T}$ stage, patients without lymph node metastasis may have a poorer OS than those with lymph node metastasis.

In clinical practice, the TNM staging system is an essential way to evaluate the prognosis of gastric patients ${ }^{24}$. However, we found that the prognosis often varies even within the same TNM stage. As Warneke stated ${ }^{25}$, the TNM staging system is a simple mathematical model, and it is difficult to reflect the actual OS without other clinicopathological factors. To assess the prognosis more 
accurately, other important clinicopathological factors should also be taken into account. As our univariate analysis results showed, age, tumor location, tumor size, and CEA are other factors associated with OS that should be considered. In the past, some studies used similar prognostic risk factors to distinguish patients who can benefit from adjuvant chemotherapy. However, some vital clinicopathological factors were unbalanced, or the number of patients from a single center was relatively small ${ }^{17,26}$.

There is no denying that age must be a key factor in prognosis. Research in the Netherlands has confirmed this association ${ }^{27}$. Toru Aoyama et al. ${ }^{28}$ and Pompiliu Piso et al. ${ }^{29}$ reported that the long-term survival of patients with distal tumors was more satisfactory than that of patients with proximal tumors. There is a high proportion of undifferentiated tumors in the proximal stomach, which tend to have a poorer prognosis than differentiated tumors. Anatomically, the intra-abdominal part of the cardia and fundus are not fully covered by visceral peritoneum, so proximal gastric cancer is more likely to infiltrate the serosa and more prone to peritoneal metastasis ${ }^{30}$. In terms of surgery, radical surgery for proximal gastric cancer is D2 total gastrectomy, which is more invasive than surgery for distal gastric cancer ${ }^{28}$. Linitis plastica has the poorest prognosis among all tumor locations. In Asia, linitis plastica is defined as Borrmann IV gastric cancer ${ }^{15}$. A recent study showed that linitis plastica has a high risk for peritoneal involvement $(75.2 \%$ among patients with radiographically nonmetastatic disease), so linitis plastica was an independent prognostic 
factor $^{31}$. In the past, some studies showed that a larger tumor size may increase the difficulty of surgery or be associated with a more advanced Borrmann type, deeper depth of invasion, and higher incidence of lymph node metastases, all of which contribute to poorer $\mathrm{OS}^{32,33}$.

There are some limitations in our study. First, this study is a retrospective study, so selection bias is inevitable. However, we used PSM to adjust the baseline characteristics and reduce the influence of selection bias. Second, considering the large number of cases from multiple centers in this study, our results may be generalized, at least in Asia, but these results need to be further validated in European and American cohorts because all patients in this study were from China. Third, the adjuvant chemotherapy regimens were uniform.

\section{Conclusion}

In conclusion, adjuvant chemotherapy can improve OS in stage II gastric cancer patients. Tumor size, CEA, and $\mathrm{N}$ stage are prognostic factors, and age, tumor location, and T stage are independent prognostic factors, thus providing significant evidence for doctors to identify patients with poor prognosis and helping guide individual therapy.

References

1. Sitarz R, Skierucha M, Mielko J, Offerhaus GJA, Maciejewski R, 
Polkowski WP. Gastric cancer: epidemiology, prevention, classification, and treatment. Cancer Manag Res. 2018;10:239-248.

2. Fock KM. Review article: the epidemiology and prevention of gastric cancer. Aliment Pharmacol Ther. 2014;40(3):250-260.

3. Sano T. Gastric cancer: Asia and the world. Gastric Cancer. 2017;20(Suppl 1):1-2.

4. Wang F-H, Shen L, Li J, et al. The Chinese Society of Clinical Oncology (CSCO): clinical guidelines for the diagnosis and treatment of gastric cancer. Cancer Commun (Lond). 2019;39(1):10-10.

5. Sasako M, Sakuramoto S Fau - Katai H, Katai H Fau - Kinoshita T, et al. Five-year outcomes of a randomized phase III trial comparing adjuvant chemotherapy with S-1 versus surgery alone in stage II or III gastric cancer. (1527-7755 (Electronic)).

6. Noh SH, Park SR, Yang H-K, et al. Adjuvant capecitabine plus oxaliplatin for gastric cancer after D2 gastrectomy (CLASSIC): 5-year follow-up of an open-label, randomised phase 3 trial. Lancet Oncol. 
2014;15(12):1389-1396.

7. Bang YJ, Kim Yw Fau - Yang H-K, Yang Hk Fau - Chung HC, et al. Adjuvant capecitabine and oxaliplatin for gastric cancer after D2 gastrectomy (CLASSIC): a phase 3 open-label, randomised controlled trial. (1474-547X (Electronic)).

8. Jiang Y, Li T, Liang X, et al. Association of Adjuvant Chemotherapy With Survival in Patients With Stage II or III Gastric Cancer. JAMA Surg. 2017;152(7):e171087-e171087.

9. Choi YY, Cheong J-H. Comment on "To Treat, or Not to Treat, That is the Question: Biomarker-guided Adjuvant Chemotherapy for Stage II and III Gastric Cancer". Ann Surg. 2019;270(2):e40-e41.

10. Torre LA, Bray F Fau - Siegel RL, Siegel RI Fau - Ferlay J, Ferlay J Fau - Lortet-Tieulent J, Lortet-Tieulent J Fau - Jemal A, Jemal A. Global cancer statistics, 2012. (1542-4863 (Electronic)).

11. Macdonald JS, Smalley Sr Fau - Benedetti J, Benedetti J Fau - Hundahl SA, et al. Chemoradiotherapy after surgery compared with surgery alone 
for adenocarcinoma of the stomach or gastroesophageal junction. (0028-4793 (Print)).

12. Cunningham D, Allum Wh Fau - Stenning SP, Stenning Sp Fau Thompson JN, et al. Perioperative chemotherapy versus surgery alone for resectable gastroesophageal cancer. (1533-4406 (Electronic)).

13. Sakuramoto S, Sasako M Fau - Yamaguchi T, Yamaguchi T Fau Kinoshita T, et al. Adjuvant chemotherapy for gastric cancer with S-1, an oral fluoropyrimidine. (1533-4406 (Electronic)).

14. Sasako M, Inoue M Fau - Lin J-T, Lin Jt Fau - Khor C, Khor C Fau - Yang H-K, Yang Hk Fau - Ohtsu A, Ohtsu A. Gastric Cancer Working Group report. (1465-3621 (Electronic)).

15. Japanese Gastric Cancer A. Japanese gastric cancer treatment guidelines 2014 (ver. 4). Gastric Cancer. 2017;20(1):1-19.

16. Nakajima T, Nashimoto A Fau - Kitamura M, Kitamura M Fau - Kito T, et al. Adjuvant mitomycin and fluorouracil followed by oral uracil plus tegafur in serosa-negative gastric cancer: a randomised trial. Gastric 
Cancer Surgical Study Group. (0140-6736 (Print)).

17. Yura M, Yoshikawa TA-O, Otsuki S, et al. Is surgery alone sufficient for treating $\mathrm{T} 1$ gastric cancer with extensive lymph node metastases? (1436-3305 (Electronic)).

18. Nashimoto A, Nakajima T Fau - Furukawa H, Furukawa H Fau Kitamura $\mathrm{M}$, et al. Randomized trial of adjuvant chemotherapy with mitomycin, Fluorouracil, and Cytosine arabinoside followed by oral Fluorouracil in serosa-negative gastric cancer: Japan Clinical Oncology Group 9206-1. (0732-183X (Print)).

19. Xiao $\mathrm{H}$, Zhou $\mathrm{H}$, Zhang $\mathrm{P}$, et al. Association among the prognostic nutritional index, completion of adjuvant chemotherapy, and cancerspecific survival after curative resection of stage II/III gastric cancer. Eur J Clin Nutr. 2019:10.1038/s41430-41019-40502-41431.

20. Jiang Y, Zhang Q, Hu Y, et al. ImmunoScore Signature: A Prognostic and Predictive Tool in Gastric Cancer. Ann Surg. 2018;267(3):504-513.

21. Lin J-X, Lin J-P, Xie J-W, et al. Preoperative Hematocrit (HCT) is a Novel 
and Simple Predictive Marker for Gastric Cancer Patients Who Underwent Radical Gastrectomy. Ann Surg Oncol. 2019;26(12):40274036.

22. Ghidini M, Donida BM, Totaro L, et al. Prognostic factors associated with survival in a large cohort of gastric cancer patients resected over a decade at a single Italian center: the Cremona experience. Clin Trans/ Oncol. 2019:10.1007/s12094-12019-02220-w.

23. Ding P, Gao Z, Zheng C, Chen J, Li K, Gao SA-O. Risk evaluation of splenic hilar or splenic artery lymph node metastasis and survival analysis for patients with proximal gastric cancer after curative gastrectomy: a retrospective study. (1471-2407 (Electronic)).

24. Sano T, Coit DG, Kim HH, et al. Proposal of a new stage grouping of gastric cancer for TNM classification: International Gastric Cancer Association staging project. Gastric Cancer. 2017;20(2):217-225.

25. Warneke VS, Behrens Hm Fau - Hartmann JT, Hartmann Jt Fau - Held $H$, et al. Cohort study based on the seventh edition of the TNM 
classification for gastric cancer: proposal of a new staging system. (1527-7755 (Electronic)).

26. Huang Z-N, Desiderio J, Chen Q-Y, et al. Indications for adjuvant chemotherapy in patients with AJCC stage Ila T3NOMO and T1N2M0 gastric cancer-an east and west multicenter study. BMC Gastroenterol. 2019;19(1):205-205.

27. Nelen SD, Verhoeven RHA, Lemmens VEPP, de Wilt JHW, Bosscha K. Increasing survival gap between young and elderly gastric cancer patients. Gastric Cancer. 2017;20(6):919-928.

28. Aoyama T, Yoshikawa T, Fujikawa H, et al. Prognostic factors in stage IB gastric cancer. World J Gastroenterol. 2014;20(21):6580-6585.

29. Piso P, Werner U, Lang H, Mirena P, Klempnauer J. Proximal versus distal gastric carcinoma--what are the differences? Ann Surg Oncol. 2000;7(7):520-525.

30. Wang X, Liu F, Li Y, et al. Comparison on Clinicopathological Features, Treatments and Prognosis between Proximal Gastric Cancer and Distal 
Gastric Cancer: A National Cancer Data Base Analysis. J Cancer. 2019;10(14):3145-3153.

31. Ikoma N, Agnes A, Chen H-C, et al. Linitis Plastica: a Distinct Type of Gastric Cancer. J Gastrointest Surg. 2019:10.1007/s11605-1101904422-11607.

32. Zhao LY, Zhang Wh Fau - Chen X-Z, Chen Xz Fau - Yang K, et al. Prognostic Significance of Tumor Size in 2405 Patients With Gastric Cancer: A Retrospective Cohort Study. (1536-5964 (Electronic)).

33. Shiraishi N, Inomata M Fau - Osawa N, Osawa N Fau - Yasuda K, Yasuda K Fau - Adachi Y, Adachi Y Fau - Kitano S, Kitano S. Early and late recurrence after gastrectomy for gastric carcinoma. Univariate and multivariate analyses. (0008-543X (Print)). 
TABLE1 Characteristics of Stage II Gastric Cancer Patients before and after PSM

\begin{tabular}{|c|c|c|c|c|c|c|}
\hline \multirow[t]{2}{*}{ characteristic } & \multicolumn{3}{|c|}{ Before PSM(n=1121) } & \multicolumn{3}{|c|}{ After PSM ( $n=948)$} \\
\hline & $\begin{array}{l}A C \\
{[n=805](\%)}\end{array}$ & $\begin{array}{l}\text { Surgery-only } \\
{[\mathrm{n}=316](\%)}\end{array}$ & $P$ value & $\begin{array}{l}A C \\
{[n=632](\%)}\end{array}$ & $\begin{array}{l}\text { Surgery-only } \\
{[\mathrm{n}=316](\%)}\end{array}$ & y $P$ value \\
\hline Age & & & 0.002 & & & 1.000 \\
\hline$<60$ & $415(51.6)$ & $130(41.1)$ & & $260(41.1)$ & $130(41.1)$ & \\
\hline$\geqq 60$ & $390(48.4)$ & 186(58.9) & & $372(58.9)$ & $186(58.9)$ & \\
\hline Sex & & & 0.102 & & & 0.255 \\
\hline male & $567(70.4)$ & $238(75.3)$ & & $454(71.8)$ & $238(75.3)$ & \\
\hline female & $238(29.6)$ & $78(24.7)$ & & $178(28.2)$ & $78(24.7)$ & \\
\hline Tumor location & & & 0.143 & & & 0.296 \\
\hline upper & $236(29.3)$ & $106(33.5)$ & & $201(31.8)$ & $106(33.5)$ & \\
\hline middle & $139(17.3)$ & $44(24.0)$ & & $105(16.6)$ & $44(13.9)$ & \\
\hline lower & $383(47.6)$ & 155(49.1) & & $290(45.9)$ & $155(49.1)$ & \\
\hline linitis plastica & $47(5.8)$ & $11(3.5)$ & & $36(5.7)$ & $11(3.5)$ & \\
\hline Tumor size & & & 0.638 & & & 0.849 \\
\hline$<5 \mathrm{~cm}$ & $519(64.5)$ & 199(63.0) & & $402(63.6)$ & 199(63.0) & \\
\hline$\geqq 5 \mathrm{~cm}$ & $286(35.5)$ & $117(37.0)$ & & $230(36.4)$ & $177(37.0)$ & \\
\hline SCA & & & 0.007 & & & 0.038 \\
\hline yes & $145(18.0)$ & $36(11.4)$ & & $104(16.5)$ & $36(11.4)$ & \\
\hline no & $660(82.0)$ & $280(88.6)$ & & $528(83.5)$ & $280(88.6)$ & \\
\hline MCA & & & 0.193 & & & 0.240 \\
\hline yes & $69(8.6)$ & $35(11.1)$ & & $55(8.7)$ & $35(11.1)$ & \\
\hline no & $736(91.4)$ & 281(88.9) & & $577(91.3)$ & 281(88.9) & \\
\hline CA199(U/ml) & & & 0.623 & & & 0.436 \\
\hline$<37$ & $710(88.2)$ & $282(89.2)$ & & $553(87.5)$ & $282(89.2)$ & \\
\hline$\geqq 37$ & $95(11.8)$ & $34(10.8)$ & & $79(12.5)$ & $34(10.8)$ & \\
\hline $\mathrm{CEA}(\mathrm{ng} / \mathrm{ml})$ & & & 0.627 & & & 0.951 \\
\hline
\end{tabular}




\begin{tabular}{lllllll}
$<5$ & $677(84.1)$ & $262(82.9)$ & & $525(83.1)$ & $262(82.9)$ & \\
$\geqq 5$ & $128(15.9)$ & $54(17.1)$ & & $107(16.9)$ & $54(17.1)$ & \\
$\mathrm{HB}(\mathrm{g} / \mathrm{L})$ & & & 0.297 & & & 0.166 \\
$<120$ & $289(35.9)$ & $124(39.2)$ & & $219(34.7)$ & $124(39.2)$ & \\
$\geqq 120$ & $516(64.1)$ & $192(60.8)$ & & $413(65.3)$ & $192(60.8)$ & \\
T stage & & & 0.086 & & & 0.088 \\
T1/2/3 & $665(82.6)$ & $247(78.2)$ & & $523(82.8)$ & $247(78.2)$ & \\
T4 & $140(17.4)$ & $69(21.8)$ & & $109(17.2)$ & $69(21.8)$ & \\
N stage & & & 0.141 & & & 0.215 \\
positive & $424(52.7)$ & $151(47.8)$ & & $329(52.1)$ & $151(47.8)$ & \\
negative & $381(47.3)$ & $165(52.2)$ & & $303(47.9)$ & $165(52.2)$ & \\
\hline
\end{tabular}

SCA signet ring cell carcinoma; MCA mucinous adenocarcinoma; CEA carcinoembryonic antigen; CA199 carbohydrate antigen 19-9

TABLE2 Univariate Analysis of Factors Associated with Overall Survival before and after PSM

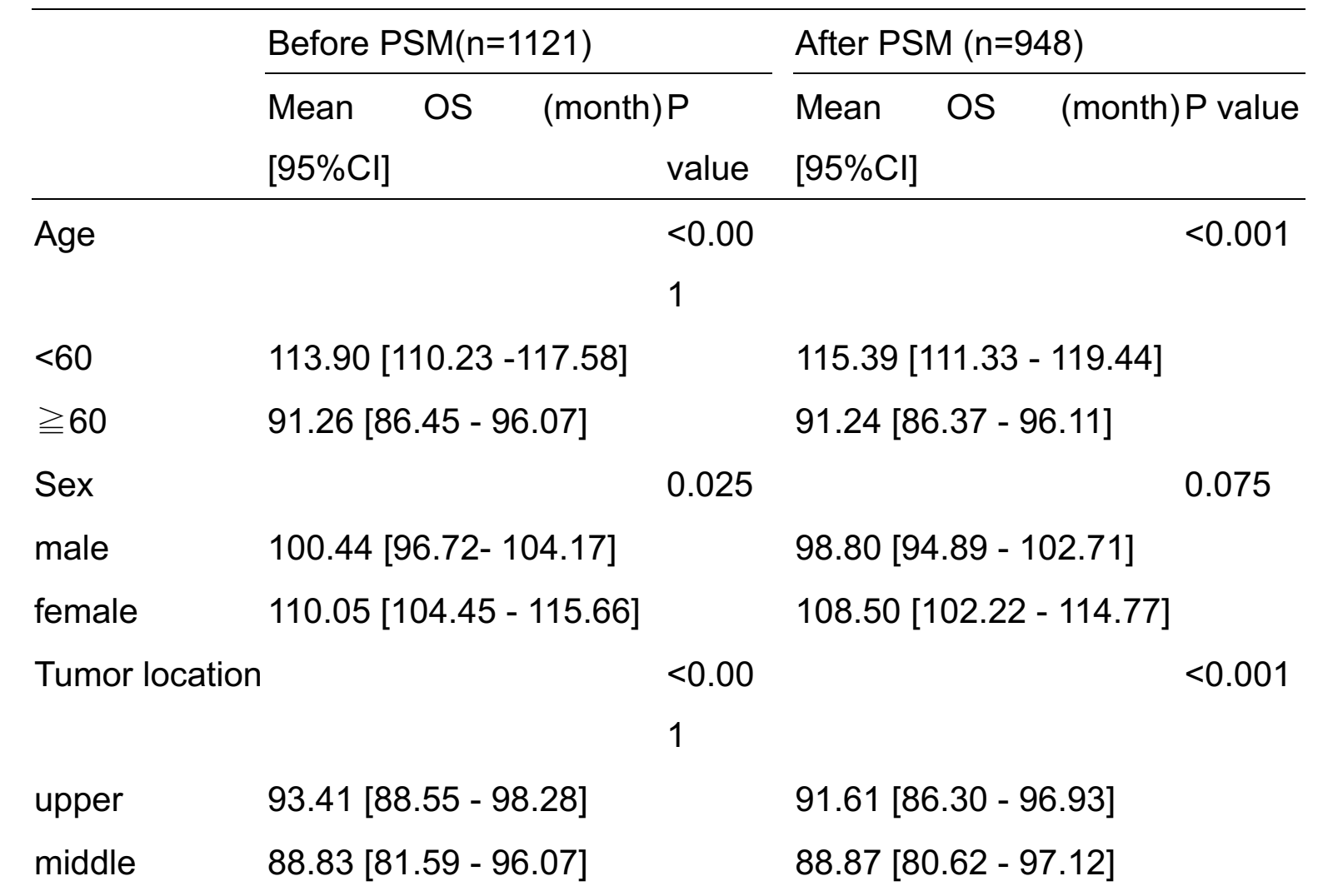




\begin{tabular}{|c|c|c|c|c|}
\hline lower & 110.89 [106.79 - 114.98] & & 111.21 [106.80-115.63] & \\
\hline linitis plastica & $63.26[51.61-74.91]$ & & 62.28 [49.97 - 74.59] & \\
\hline \multirow[t]{2}{*}{ Tumor size } & & $<0.00$ & & 0.002 \\
\hline & & 1 & & \\
\hline$<5 \mathrm{~cm}$ & $109.01[105.20$ - 112.83] & & $107.99[103.72-112.27]$ & \\
\hline \multirow[t]{2}{*}{$\geqq 5 \mathrm{~cm}$} & $95.76[90.16-$ & & $89.71[84.49-94.93]$ & \\
\hline & 101.37] & & & \\
\hline SCA & & 0.164 & & 0.122 \\
\hline yes & $111.02[104.65$ - 117.38] & & $111.35[104.17$ - 118.52] & \\
\hline no & $101.11[97.61$ - 104.60] & & 97.42 [93.83 - 101.02] & \\
\hline MCA & & 0.740 & & 0.622 \\
\hline yes & 89.21 [82.56 - 95.87] & & 89.67 [82.71 - 96.63] & \\
\hline no & 104.98 [101.57 - 108.40] & & $104.22[100.48-107.96]$ & \\
\hline CA199(U/ml) & & 0.143 & & 0.093 \\
\hline$<37$ & $106.21[102.86$ - 109.56] & & $105.86[102.22-109.50]$ & \\
\hline$\geqq 37$ & 82.17 [74.61 - 89.74] & & 80.08 [71.58 - 88.58] & \\
\hline $\mathrm{CEA}(\mathrm{ng} / \mathrm{ml})$ & & 0.014 & & 0.010 \\
\hline$<5$ & $106.67[103.22$ - 110.12] & & 106.31 [102.54 - 110.08] & \\
\hline$\geqq 5$ & 78.17 [72.40 - 83.93] & & $77.03[70.80$ - 83.25] & \\
\hline $\mathrm{HB}(\mathrm{g} / \mathrm{L})$ & & 0.206 & & 0.140 \\
\hline$<120$ & 99.08 [94.01 - 104.14] & & 97.49 [91.83 - 103.15] & \\
\hline$\geqq 120$ & $106.34[102.36-110.32]$ & & $106.21[101.91-110.50]$ & \\
\hline T stage & & 0.009 & & 0.013 \\
\hline T123 & $103.16[99.89-106.43]$ & & 102.45 [98.81 - 106.09] & \\
\hline T4 & 97.99 [91.00 - 104.97] & & $97.02[89.34-104.70]$ & \\
\hline $\mathrm{N}$ stage & & 0.006 & & 0.035 \\
\hline positive & $106.01[102.45$ - 109.57] & & $104.76[100.73-108.80]$ & \\
\hline negative & 99.95 [94.99 - 104.90] & & 100.08 [94.77 - 105.40] & \\
\hline
\end{tabular}

CEA carcinoembryonic antigen; CA199 carbohydrate antigen 19-9 
TABLE 3 Multivariate Cox Regression Analysis of Factors Associated with Overall Survival after PSM

\begin{tabular}{lll}
\hline & \multicolumn{2}{l}{ Overall survival } \\
\cline { 2 - 3 } & HR[95\%Cl] & P value \\
\hline Age & & \\
$<60$ & Reference & \\
$\geqq 60$ & $2.953[1.959-4.452]$ & $<0.001$ \\
Tumor location & & \\
upper & Reference & \\
middle & $0.867[0.523-1.437]$ & 0.579 \\
lower & $0.619[0.416-0.920]$ & 0.018 \\
linitis plastica & $2.647[1.528-4.585]$ & 0.001 \\
T stage & & \\
T123 & Reference & \\
T4 & $1.667[1.148-2.421]$ & 0.007 \\
Adjuvant & & \\
chemotherapy & & \\
no & Reference & \\
yes & $0.683[0.485-0.964]$ & 0.030 \\
\hline HR hazard ratio; Cl confidence interval &
\end{tabular}


a

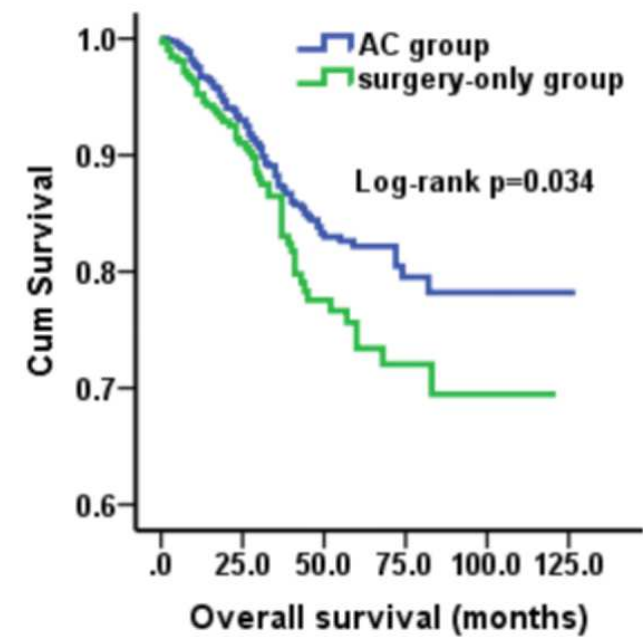

b

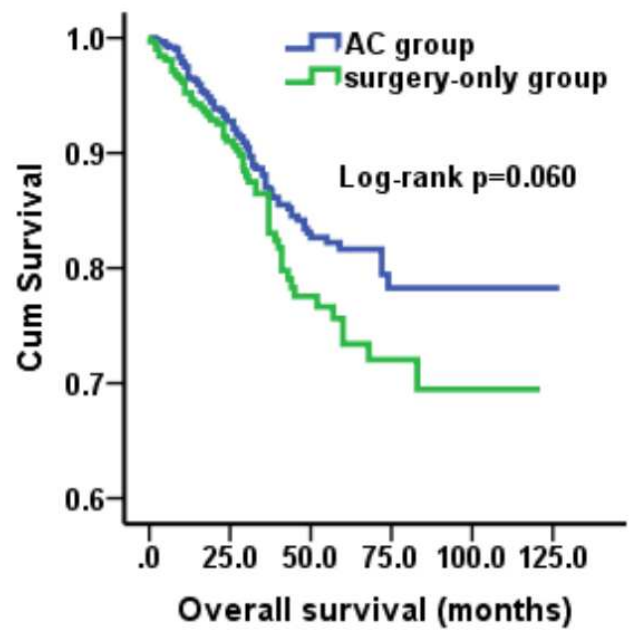

Fig.1 Kaplan-Meier analysis of overall survival in adjuvant chemotherapy group and surgery-only group. a before PSM ( $p=0.034)$; $\mathbf{b}$ after PSM $(p=0.060)$.

a

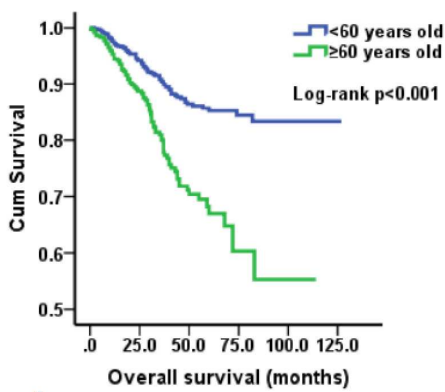

d

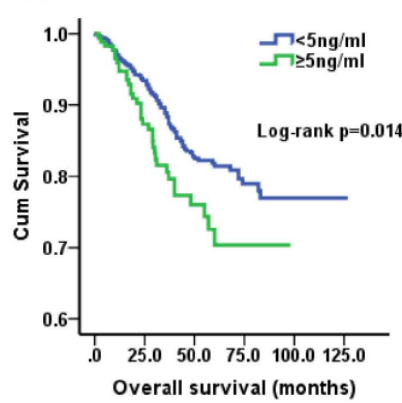

b

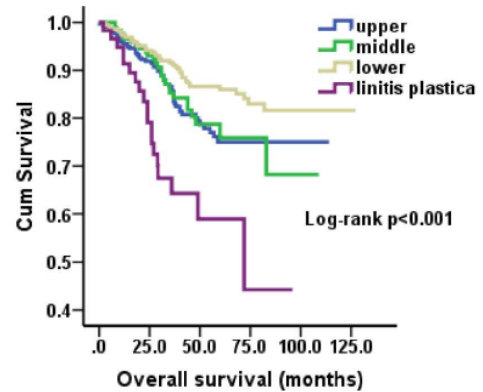

e

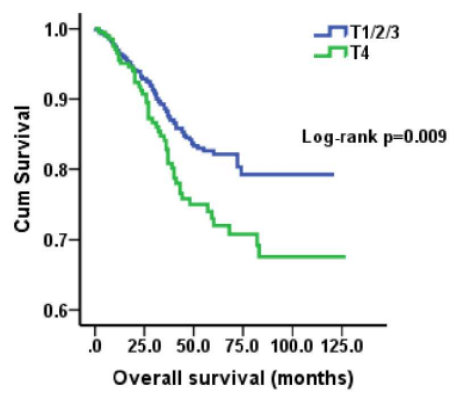

C

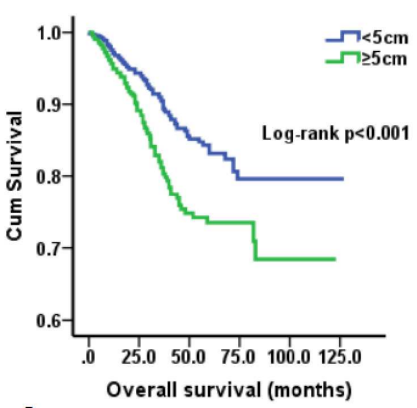

f

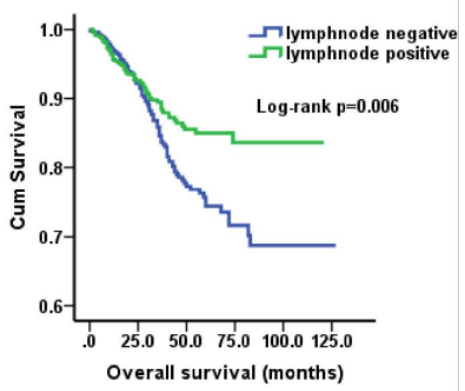

Fig.2 Kaplan-Meier survival curves of overall survival before PSM. a age, $p<0.001$; $b$ Tumor location, $p<0.001$; Tumor size, $p<0.001$; d CEA, $p=0.014 ; \mathbf{e}$ T stage, $p=0.009 ; \mathbf{f} \mathrm{N}$ stage, $\mathrm{p}=0.006$. 
a

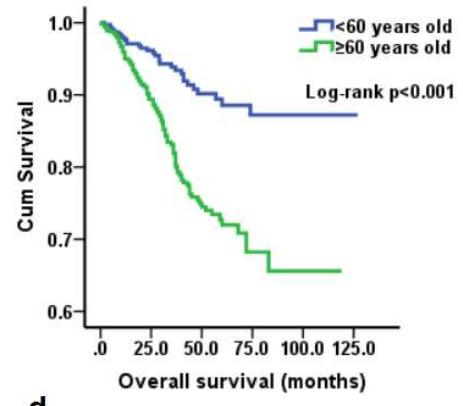

d

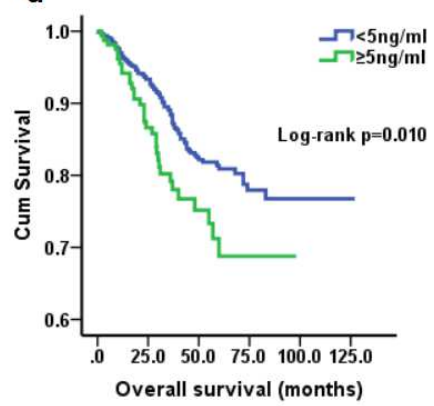

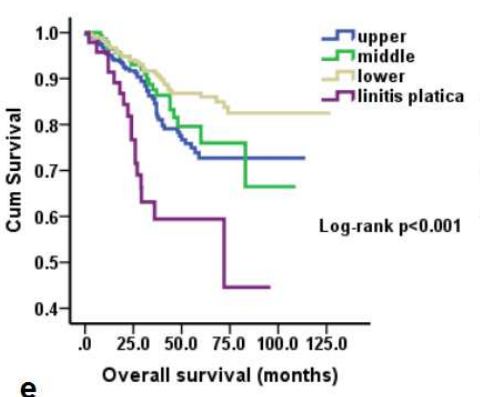

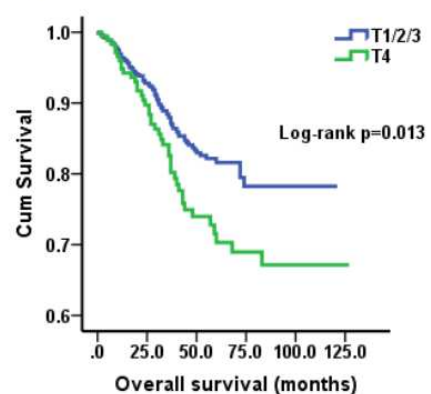

c

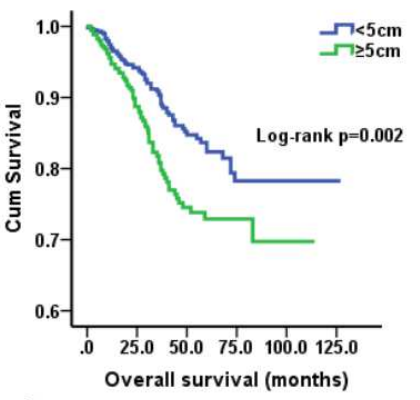

f

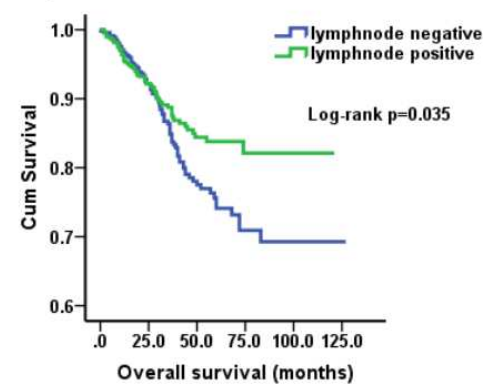

Fig.3 Kaplan-Meier survival curves of overall survival after PSM. a age, $p<0.001 ; \mathbf{b}$ Tumor location, $p<0.001$; $\mathbf{c}$ Tumor size, $p<0.002$; d CEA, $p=0.010 ; \mathbf{e}$ Tstage, $p=0.013 ; \mathbf{f} \mathrm{N}$ stage, $p=0.035$.

a

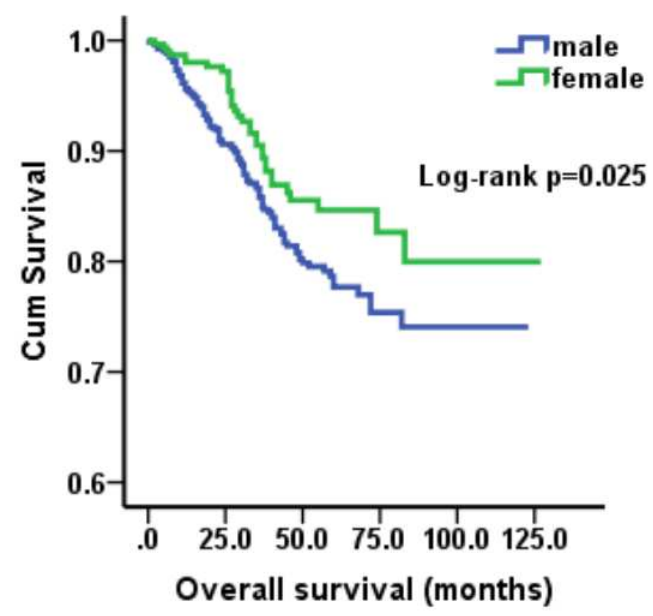

b

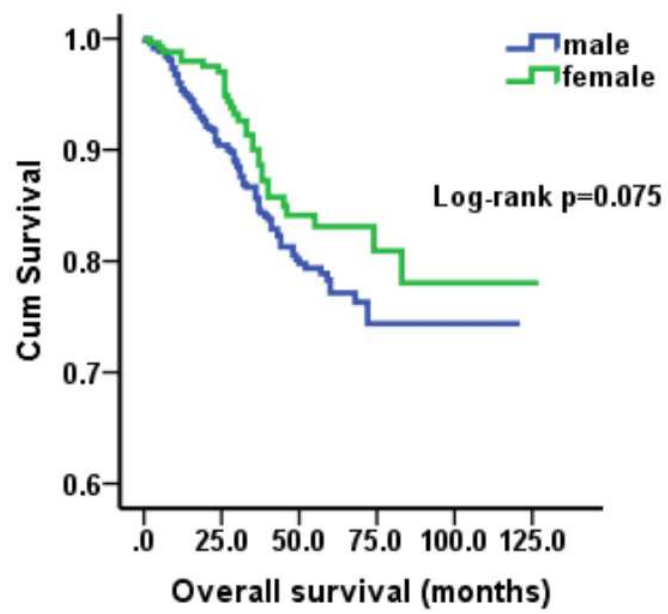

Fig.4 Kaplan-Meier survival curves of overall survival in sex. a before PSM $(p=0.025)$; $b$ after PSM $(p=0.075)$. 


\section{Declarations}

\section{Ethics approval and consent to participate}

This study was conducted in accordance with the ethical standards of the World Medical Association Declaration of Helsinki and the Ethical Guidelines for Clinical Research. The protocol was approved by the ethics committee of Sixth affiliated hospital of Sun Yat-sen University; Fujian Medical University Union Hospital and Sun Yat-sen University cancer center. Informed consent was provided by all patients.

\section{Consent for publication}

Not applicable

\section{Availability of data and materials}

The datasets used and/or analysed during the current study are available from the corresponding author on reasonable request.

\section{Competing interests}

The authors declare that they have no competing interests.

\section{Funding}

Supported by Guangzhou Science and Technology Project (grant number 201803010040) and Nation Key Clinical Discipline.

\section{Authors' contributions}

ZJD and RCN designed and performed the study. RCN, JL and XJC analyzed the data. ZJD, JX, and CMH collected the data. SC, YBC and JSP reviewed the study and the data. All authors read and approved the final manuscript.

\section{Acknowledgements}

Not applicable 
Figures

a

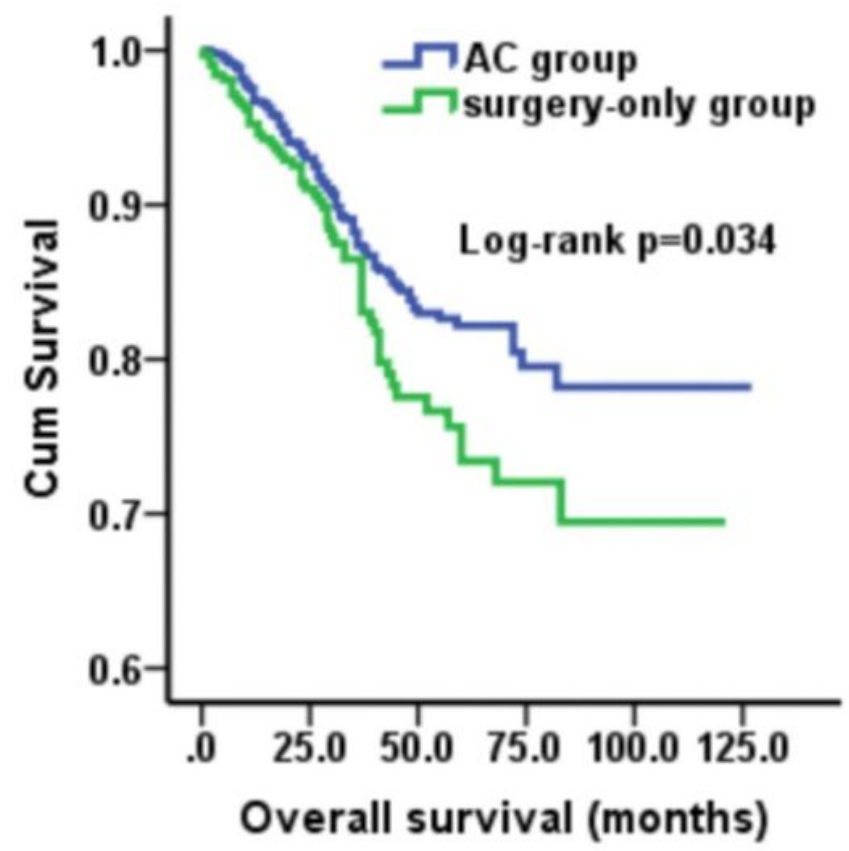

b

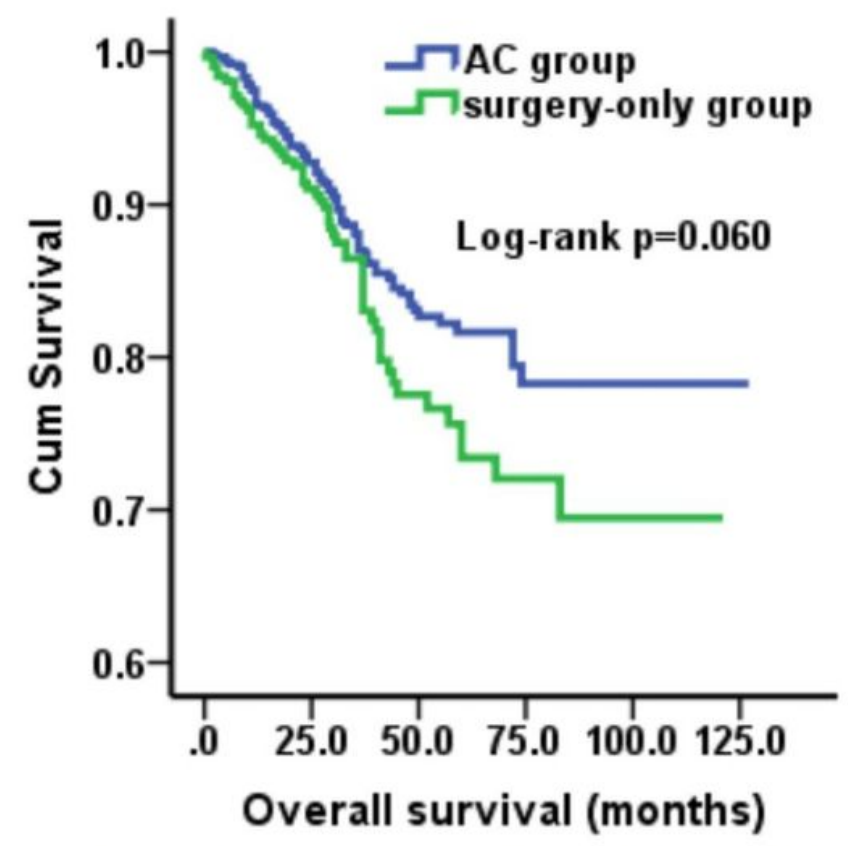

Figure 1

Kaplan-Meier analysis of overall survival in adjuvant chemotherapy group and surgery-only group. a before PSM $(p=0.034)$; $b$ after PSM $(p=0.060)$. 
a

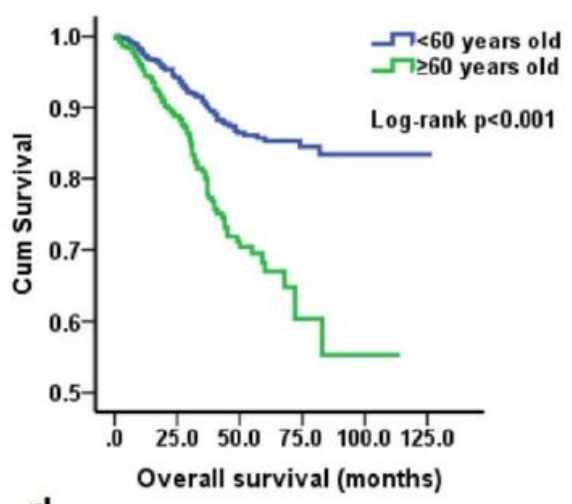

d

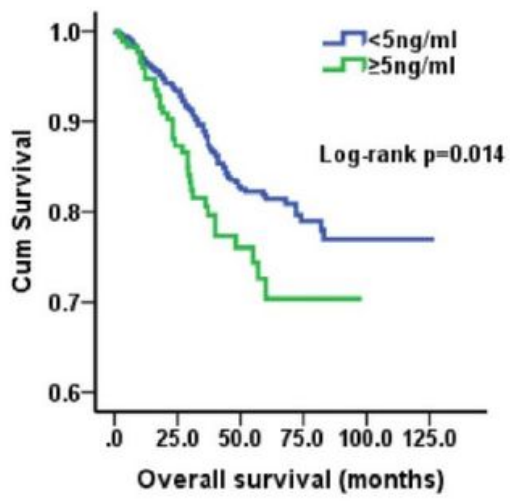

b

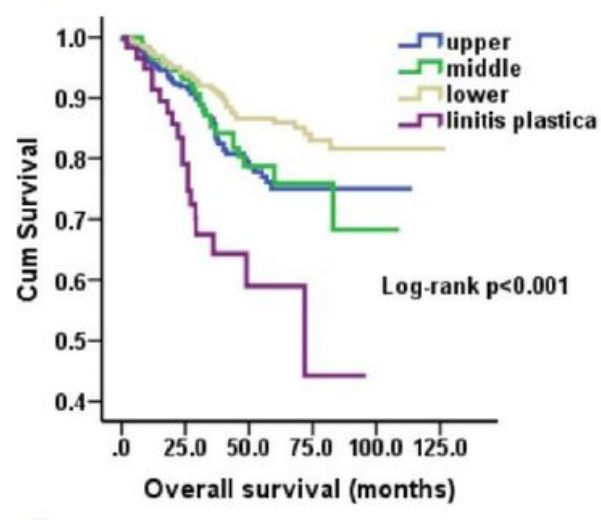

e

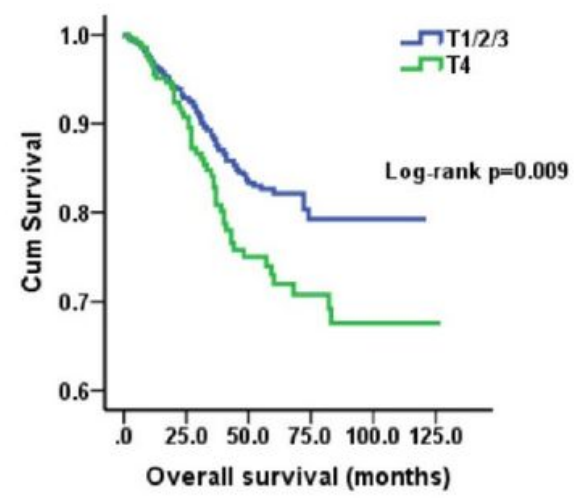

C

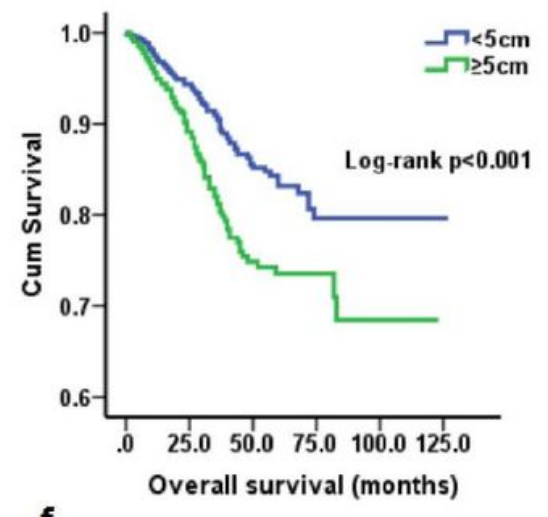

f

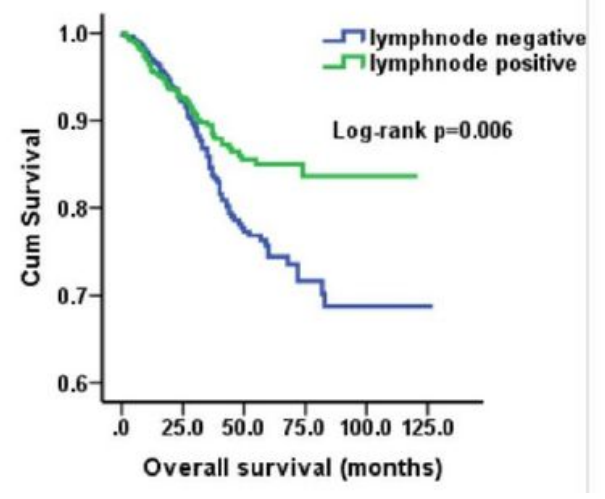

Figure 2

Kaplan-Meier survival curves of overall survival before PSM. a age, $p<0.001$; b Tumor location, $p \ll 0.001$; c Tumor size, $p<0.001$; d CEA, $p=0.014$; e T stage, $p=0.009 ; f$ N stage, $p=0.006$. 
a

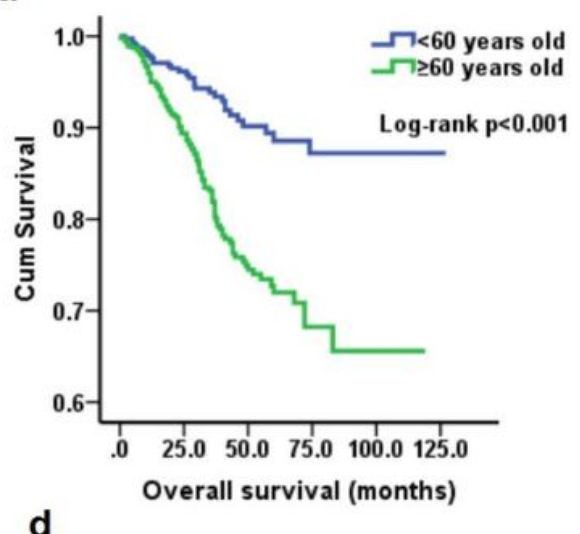

d

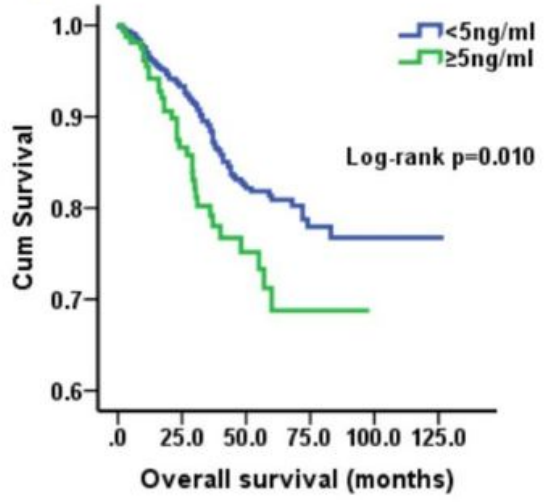

b

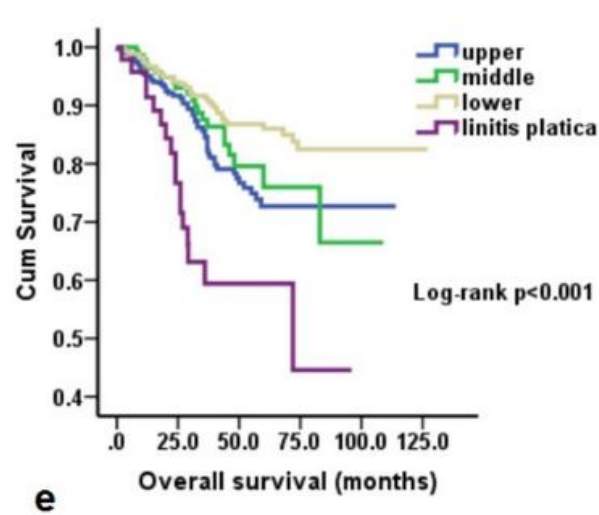

e

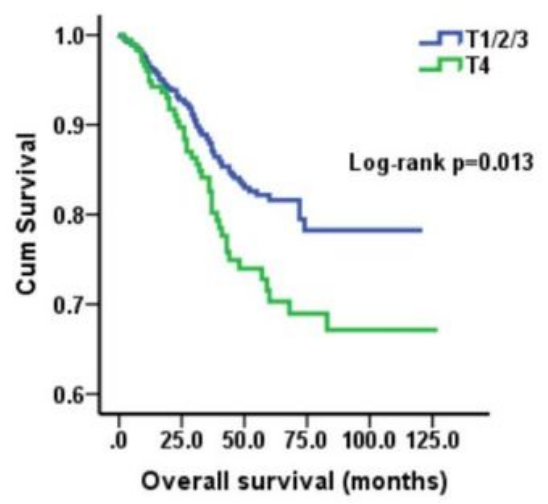

C
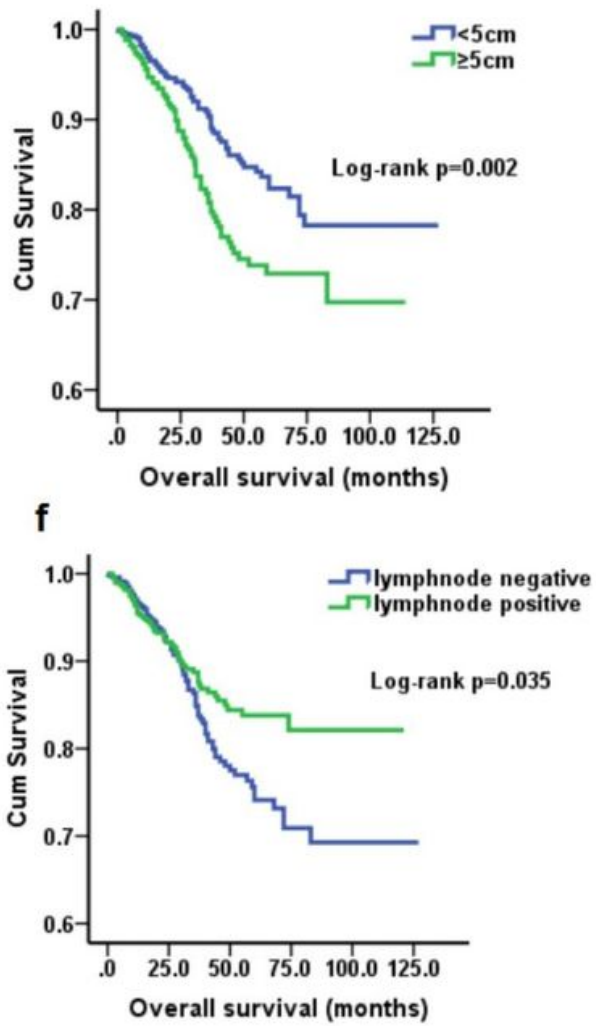

Figure 3

Kaplan-Meier survival curves of overall survival after PSM. a age, $p<0.001$; b Tumor location, $p<0.001$; c Tumor size, $p<0.002$; $d$ CEA, $p=0.010$; e Tstage, $p=0.013$; $\mathrm{N}$ stage, $p=0.035$.

a

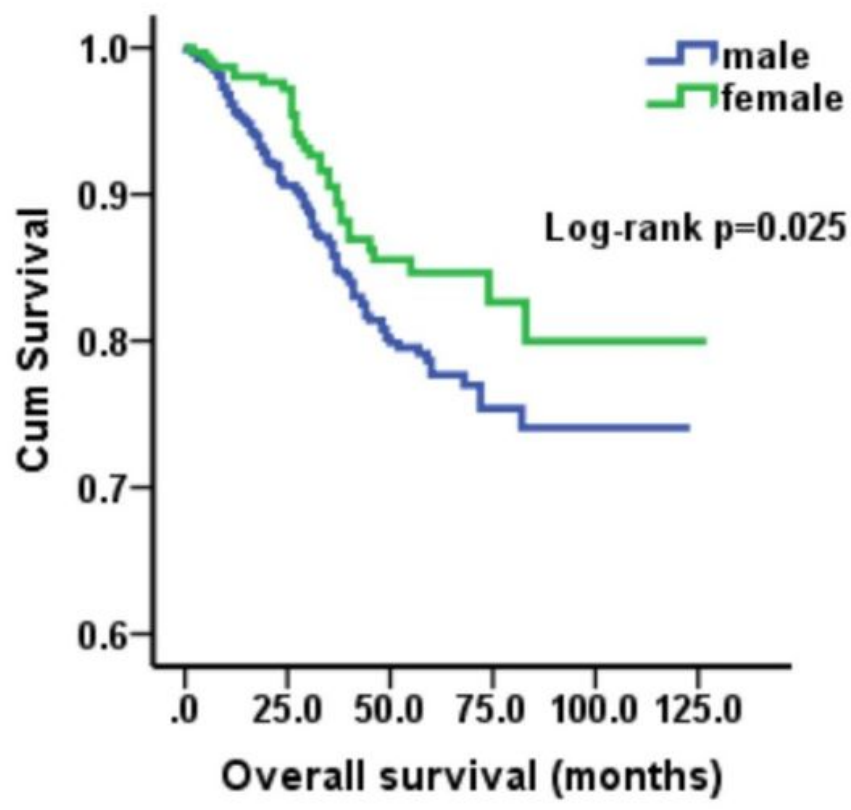

b

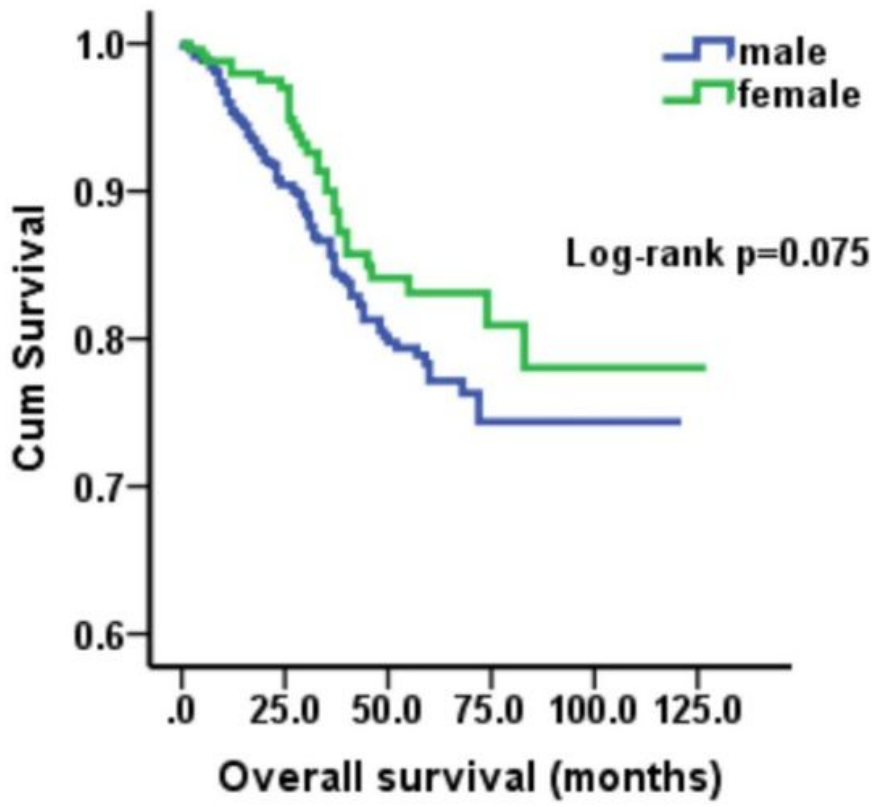

Figure 4 
Kaplan-Meier survival curves of overall survival in sex. a before PSM $(p=0.025)$; $b$ after PSM $(p=0.075)$. 